\title{
ARTICLE
}

Covid

\section{Mortality of patients with solid and haematological cancers presenting with symptoms of COVID-19 with vs without detectable SARS-COV-2: a French nationwide prospective cohort study}

\author{
Souad Assaad (1]', Philippe Zrounba' ${ }^{1}$, Claire Cropet ${ }^{2}$, ONCOVID-19 study Consortium and Jean-Yves Blay $\mathbb{D}^{1,3}$
}

\begin{abstract}
BACKGROUND: Over 30 million COVID-19 cases have been diagnosed worldwide from late 2019. Among frail persons, cancer patients are at high risk of death from COVID-19.

METHODS: The French prospective cohort ONCOVID-19 enrolled patients with solid or haematological tumour, receiving anticancer treatment and presenting with clinical symptoms suggestive of COVID-19. COVID-19 was confirmed through detectable SARS-CoV2 by RT-PCR (repeated twice if negative first) and/or specific CT-scan. The study aims to assess the 28-day mortality rate after the first COVID test.

RESULTS: From March 1st to May 21st 2020, 23 French cancer centres and hospitals enrolled 1230 cancer patients with suspicion of COVID-19, including 1162 (94.5\%) matching the inclusion criteria. We identified 425 (36.6\%) COVID-19 positive patients including 155 (13.3\%) diagnosed with CT-scan only, while 737 (63.4\%) patients were COVID-19 negative. Death at day-28 occurred in $116 / 425$ (27.8\%) COVID-19 positive patients, and in 118/737 (16.3\%) COVID-19 negative patients $(p<0.0001)$. With a median follow-up of 2.1 (1.6-2.4) months, 310 (26.7\%) deaths were reported including 143 (33.6\%) in the COVID-19 positive population, and 167 (22.7\%) in the COVID-19 negative patients. Male gender, age, metastatic disease, immunosuppressive treatments, lymphopenia, COVID-19 diagnosis and diabetes were independent risk factors for death.

CONCLUSION: Patients with solid and haematological cancers presenting COVID-19 symptoms with SARS-CoV-2 RT-PCR confirmed or not are both at high-risk of early mortality. COVID-19 is reported as the cause of death in 50\% of COVID-19 positive patients with cancer.
\end{abstract}

CLINICAL TRIAL REGISTRATION: This trial is registered with ClinicalTrials.gov, number NCT04363632.

British Journal of Cancer (2021) 125:658-671; https://doi.org/10.1038/s41416-021-01452-4

\begin{abstract}
BACKGROUND
Over 30 million cases of COVID-19 have been diagnosed worldwide, and across different countries, mortality rates range from 0.3 to $10 \%$ of cases confirmed, depending on local virology testing policies. ${ }^{1,2}$ Epidemiological studies show that the mortality rate of COVID-19 is higher in frail persons. Older adults are at greater risk of death or hospitalisation if diagnosed with COVID-19. Eight out of 10 COVID-19 deaths reported in the United States have been reported in adults 65 years old and older. ${ }^{3}$ The most common medical comorbidities were hypertension (16\%), cardiovascular disease (12\%) and diabetes (10\%). ${ }^{4}$ Cancer patients, including patients with active anticancer treatment or recovering from cancer, are a highly vulnerable group at high risk of severe clinical events and lethal complications of COVID-19. ${ }^{5-18}$ In most series, cancer patients with documented COVID-19 have a dismal
\end{abstract}

prognosis with $60-80 \%$ 30-day mortality. ${ }^{12-18}$ The retrospective study PRE-ONCOVID-19 conducted in over 400 cancer patients previously reported a 30-day mortality of $20 \%$ in patients with COVID-19 symptoms with or without detectable SARS-COV-2 on RT-PCR. ${ }^{18}$ Other viral infections, such as seasonal influenza, Influenza A H1N1 and SARS-CoV-1, were associated with high mortality rates, often $>15 \%$ in patients with cancer or immunosuppressed populations, in particular in patients with haematologic malignancies and pneumonia. ${ }^{19-21}$

The diagnosis of COVID-19 remains challenging both for viral diagnosis using RT-PCR and for serological testing. ${ }^{22,23}$ Patients with cancer were reported to have a lower rate of seroconversion after documented infection. ${ }^{24} \mathrm{CT}$-chest imaging may detect a high proportion of COVID-19-infected patients who were negative on RT-PCR testing. ${ }^{25}$

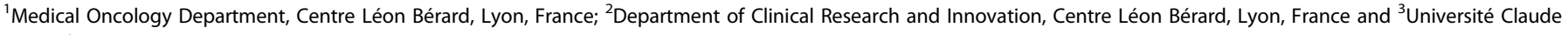
Bernard, Lyon, France \& Unicancer, Paris, France

Correspondence: Souad Assaad (souad.assaad@lyon.unicancer.fr) or Jean-Yves Blay (jean-yves.blay@lyon.unicancer.fr)

A full list of members and their affiliations appears in the Supplementary Information.

Received: 23 December 2020 Revised: 26 April 2021 Accepted: 27 May 2021

Published online: 16 June 2021 
This French multicentric study enrolled patients with cancer presenting with suspicion of COVID-19 in one of the authorised investigation sites, for whom a documented COVID-19 diagnosis (based on RT-PCR test or chest CT scan) was obtained. Cancer patients were identified as COVID-19 positive (i.e. SARS-COV-2 RT-PCR positive or CT-scan positive) or COVID-19 negative (i.e. SARS-COV-2 RT-PCR and CT-scan-negative) patients. The primary objective was to describe the characteristics and outcome of cancer patients, suspected to be infected by COVID-19, with documented COVID-19-positive diagnosis (SARS-COV-2 RT-PCR+ or COVID-19-specific chest CT scan), or COVID-19- negative diagnosis.

\section{METHODS}

Patients

This French academic multicentre observational prospective study included all patients with cancer, aged $\geq 18$ years, showing symptoms suspicious for COVID-19 disease, attending one of the participating centres for consultation appointment or hospitalised from March 1st 2020 to May 21st 2020 and tested for COVID-19, who were eligible. Patients had a confirmed diagnosis of any type of solid or haematologic tumour, and had received an anticancer curative treatment (cytotoxics, targeted treatment, immunotherapy and locoregional procedures) within the last 3 months prior to suspicion of COVID-19 infection, and had documented COVID-19 disease. Patients with febrile neutropenia were included. No exclusion criteria were specified. This trial is registered with ClinicalTrials.gov, number NCT04363632.

\section{Centres}

Twenty-three Comprehensive Cancer Centers from UNICANCER university and general hospitals participated in this research (Supplementary Table S1). Local approval from the Data Protection Officer of the sponsor centre Léon Bérard was obtained in accordance with French national and European laws and directives. The coordinating centre complies with the MR-004 French data protection authority (CNIL) requirements (project reference: R201004-040). Patients received a patient information sheet, and were offered the possibility to accept or refuse the participation to this non-interventional study. Prospective enrolment occurred at each investigational site as soon as authorised.

\section{Procedures}

From March 1st 2020 to May 21st 2020, the study was proposed to all patients from the participating institutions tested for COVID-19 disease, with a positive or a negative result. Tests for COVID-19 were performed at each investigational site. The COVID-19 diagnosis used local RT-PCR testing and/or identified specific signs of COVID-19 on CT-scan chest imaging i.e. ground-glass opacity, and consolidative opacities (49-94\% and 11-73\%, respectively, reported in previous literature), interlobular septal thickening, pleural thickening and bronchiectasis. Regarding lesion distribution, involvement of multiple lobes of both lungs was common (65-97\%). Peripheral zone and lower lobes were more predisposed to be affected. ${ }^{25,26}$ In this pandemic context, centres engaged their best efforts to comply to the national recommendations in this epidemic context, and locally organised reviews by experimented pneumologists and radiologists, but no central review has been performed. All patients with respiratory symptoms were tested by CT scan. Patients with COVID-19 clinical symptoms and initial COVID-19-negative diagnosis by RT-PCR had another RT-PCR test within the next 7 days; the date of initial COVID-19 diagnosis procedure was retained in the case of results confirmed as negative, and the second testing date was retained for COVID-19-positive patients. SARS-CoV-2 RT-PCR diagnosis tests were performed on nasopharyngeal swabs using the cobas ${ }^{\circledR}$ SARSCoV-2 Test (Roche, Neuilly, France) exploring ORF1/a and Pan
SARS gene E. For each patient, a follow-up at 28 days after the COVID-19 diagnosis test was performed and included seven category scales and vital status updated once for all patients 28 days after the last patient accrued. Each investigational site reported patient data in a secure database. Quality control of data was performed using automatic data controls and remote monitoring.

COVID-19-positive and -negative cohorts

Cancer patients with suspicion of COVID-19 were identified as COVID-19 positive, based on SARS-COV-2 RT-PCR results, and in the case of two consecutive negative RT-PCR tests, diagnosis used typical CT-scan images. Cancer patients without detectable SARSCoV-2 by RT-PCR, repeated twice and without characteristic images on CT scan, were identified as COVID-19-negative patients.

Objectives of the study, patient characteristics and outcome The study aimed to describe clinical characteristics, treatment and outcome of cancer patients presenting with COVID-19 symptoms during the first pandemic wave in France. The primary endpoint of the study is the mortality of cancer patients at day 28 , defined as the proportion of deaths 28 days after COVID-19 testing. Mortality rate is presented in cancer patients with positive COVID-19 diagnosis and in patients with negative diagnosis. Secondary endpoints included (1) description of patient characteristics (demographics, tumour type, anticancer treatment, symptoms, comorbidities and laboratory parameters), (2) overall survival (OS) defined as the time from COVID-19 diagnosis test to the date of death due to any cause, (3) primary cause of death, including COVID-19-related deaths and (4) description of adverse events and treatments after COVID-19 testing. Secondary objectives included predictive factors for mortality in the global population of cancer patients with COVID-19 symptoms and in COVID-19-positive patients.

Statistics

This is a descriptive study. No sample-size calculation was therefore imposed in the epidemic context at study initiation. We nevertheless assumed that a 6-month recruitment period should allow a minimum of 150 patients to be recruited.

Qualitative variables were described using frequencies and percentages. Comparisons between groups were done using the chi-square test. Quantitative variables were described using median (Q1-Q3) and were compared between subgroups using the Wilcoxon test.

Overall survival was estimated using the Kaplan-Meier method and compared between subgroups using the Log-rank test.

Patient characteristics in the global population, in the COVID19-positive subgroup and in the COVID-19-negative subgroups, were described and analysed according to the vital status at 28 days.

The multivariate model exploring independent parameters associated with an increased risk of death at 28 days tested gender, age, history of cardiovascular disease, history of diabetes, history of autoimmune/inflammatory disease, smoker status, time from the date of cancer diagnosis to the date of COVID-19 testing, type of tumour, presence of metastatic disease, lung metastases (solid tumours), lymphopenia, time from the date of surgery to the date of the test, ongoing corticotherapy and overall COVID-19 test result (COVID-19 Positive [RT-PCR +, CT scan+]/COVID-19 Negative diagnosis). Analyses were conducted in the global population and in the COVID-19-positive population. A backward selection procedure with a cut-off $p$-value of 0.05 was used for the final multivariate model. Variables with too many missing data $(>15 \%)$ e.g. baseline CRP levels, were not included in the multivariate model. Missing values for the response or explanatory variables led to the deletion of $164(14.1 \%)$ in the global population, and 58 (13.6\%) observations in the COVID-19- positive population. 


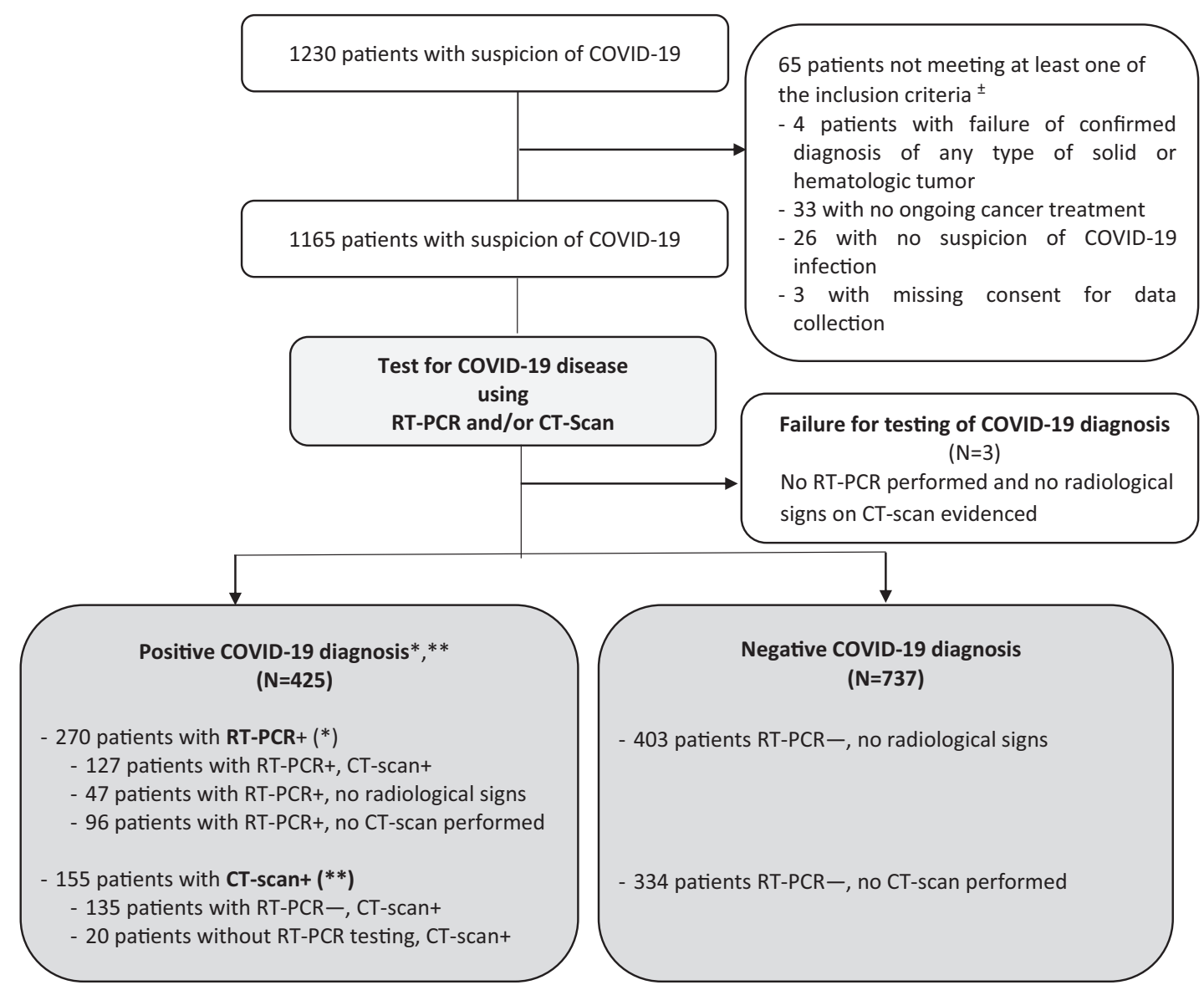

Fig. 1 Study flowchart. COVID-19 diagnosis was positive based on positive RT-PCR results ( ${ }^{*}$ RT-PCR + ), OR specific radiological signs of COVID-19 on chest CT scan ( ${ }^{* *}$ CT scan+). COVID-19 diagnosis was negative if RT-PCR result was negative AND (i) no specific radiological signs of COVID-19 on chest CT scan; (ii) no chest CT scan performed. A failure for COVID-19 diagnosis is reported in the absence of RT-PCR test and no evidence of COVID-19 radiological signs on chest CT scan. \pm Non-exclusive reasons.

The data cut-off was June 12th 2020. Statistical analyses were performed using SAS (version 9.4).

\section{RESULTS}

Description of the population

From March 1st 2020 to May 21st 2020, 23 investigational sites enrolled 1230 patients with cancer and suspected for COVID-19, including 1162 (94.5\%) cancer patients meeting criteria for eligibility, with a confirmed diagnosis of solid or haematologic cancer, any cancer treatment (SACT, RT or surgery) within the last 3 months from COVID-19 diagnosis and a documented COVID-19 diagnosis procedure (Fig. 1).

Among the 1162, 425 (36.6\%) patients had positive COVID-19 confirmed, including 270/425 (63.5\%) patients PCR+ (with or without radiological signs on CT scans), and 155/425 (36.5\%) CT+, with other infection being excluded. A total of 737 (63.4\%) patients were classified as COVID-19 negative by PCR with no radiological signs on $\mathrm{CT}$ scan or no $\mathrm{CT}$-scan imaging performed (Fig. 1).

The demographic, clinical and tumour characteristics of the global, COVID-19-positive and COVID-19-negative populations are described in Table 1. The 1162 cancer patients consulting for a suspected COVID-19 had a median age of 63 (53-72) years, 553 $(47.6 \%)$ were male. In total, $162(13.9 \%)$ had haematologic malignancies, among them $47(29 \%)$ patients had non-Hodgkin lymphomas. In total, 1000 (86.1\%) patients had solid tumours, 676 (58.2\%) had metastasis, including 294 (25.3\%) patients with lung metastasis. At the time of the COVID-19 diagnosis procedure, 700
(60.2\%) patients had received chemotherapy in the last three months, $323(27.8 \%)$ combination of cytotoxic treatment, 200 $(17.2 \%)$ had received targeted therapy and 182 immunotherapy, mostly anti-PD1 $(N=132,11.4 \%)$. In total, 658 (56.7\%) patients were receiving anticancer treatments, and 249 (21.4\%) patients were receiving corticosteroid therapy.

Subgroups of cancer patients with positive and negative COVID19 diagnosis showed few differences in baseline characteristics. There were less male patients, less metastatic patients and less patients with ongoing treatments in the COVID-19-positive group (Table 1). Patients with confirmed COVID-19-positive diagnosis identified by RT-PCR had more haematologic malignancies, and had less frequently metastatic disease (Table 1 ).

Clinical symptoms and hospitalisation in COVID-19-positive and -negative patients

The main symptoms are shown in Table 2, and included fever $(N=746,64.6 \%)$, cough $(N=495,43.3 \%)$, dyspnoea $(N=473$, $41.1 \%)$, respiratory tract infection $(N=209,18.4 \%)$ and pneumonia $(N=125,10.9 \%)$. COVID-19-positive patients had more frequently cough $(N=224,53.7 \%)$, dysgueusia $(N=38,10.5 \%)$, anosmia $(N=$ $34,9.4 \%)$, respiratory tract infection $(N=142,34.6 \%)$, pneumonia $(N=80,19.2 \%)$ and dyspnoea $(N=201,47.9 \%)$ (Supplementary Table S2A). Among the 425 COVID-19-positive patients, 412 $(96.9 \%)$ required hospitalisation, including intensive care unit for $33(10.1 \%)$ patients. The median duration of hospitalisation was 7 (1-107) days (Supplementary Table S2B). Of note, data were exclusively collected in the COVID-19-positive patients. Treatments during hospitalisation included antibiotics ( $N=221,65.4 \%)$, 
Mortality of patients with solid and haematological cancers presenting... S Assaad et al.

Table 1. Patient demographics and baseline characteristics in the global population, in the COVID-19 positive and in the COVID-19 negative groups

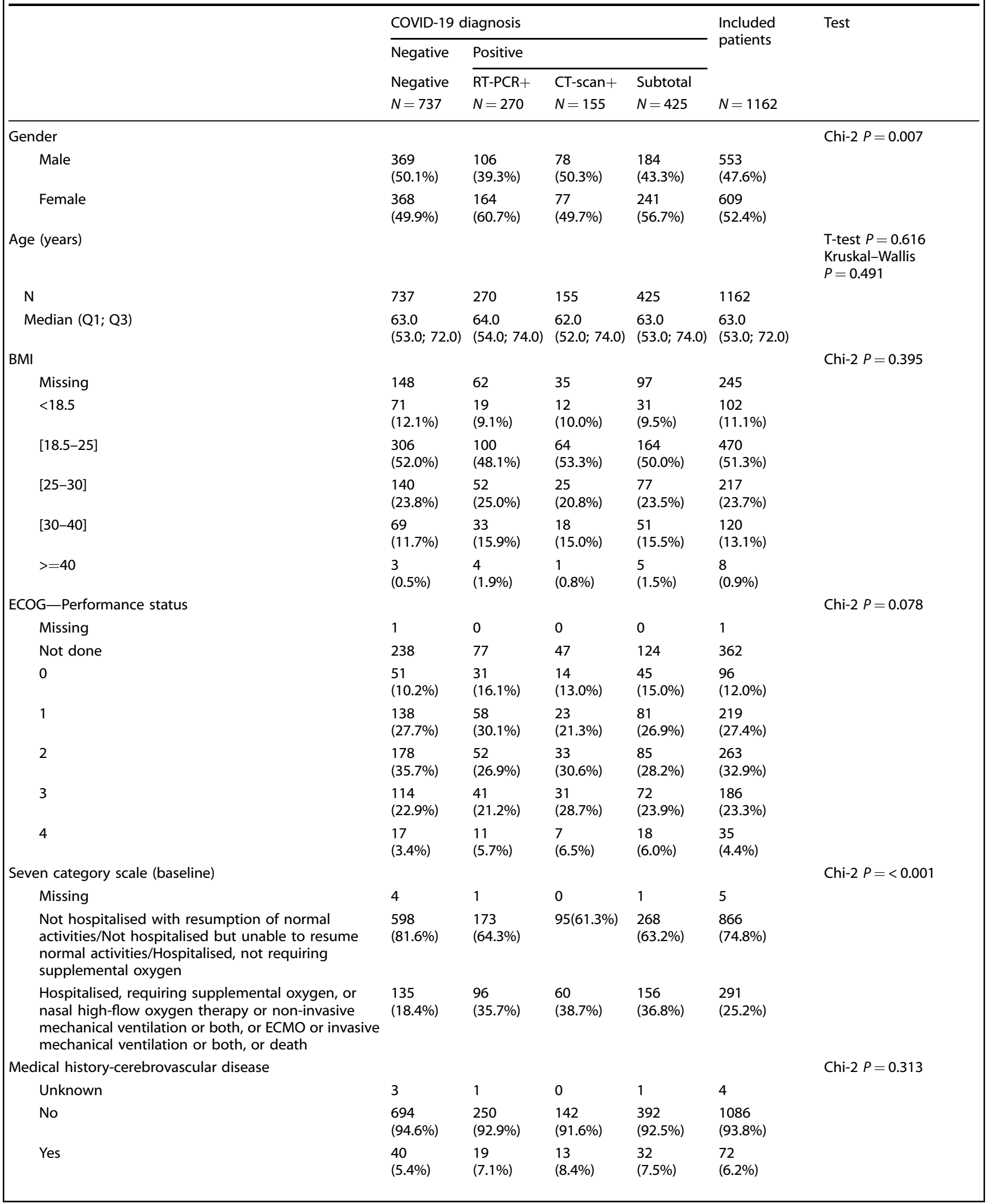


Mortality of patients with solid and haematological cancers presenting...

S Assaad et al.

Table 1. continued

\begin{tabular}{|c|c|c|c|c|c|}
\hline & \multicolumn{4}{|c|}{ COVID-19 diagnosis } & \multirow{3}{*}{$\begin{array}{l}\text { Included } \\
\text { patients } \\
N=116\end{array}$} \\
\hline & \multirow{2}{*}{$\begin{array}{l}\text { Negative } \\
\text { Negative } \\
N=737\end{array}$} & \multicolumn{3}{|l|}{ Positive } & \\
\hline & & $\begin{array}{l}\text { RT-PCR }+ \\
N=270\end{array}$ & $\begin{array}{l}\text { CT-scan+ } \\
N=155\end{array}$ & $\begin{array}{l}\text { Subtotal } \\
N=425\end{array}$ & \\
\hline \multicolumn{6}{|c|}{ Cardiovascular disease } \\
\hline Unknown & 1 & 0 & 0 & 0 & 1 \\
\hline No & $\begin{array}{l}400 \\
(54.3 \%)\end{array}$ & $\begin{array}{l}143 \\
(53.0 \%)\end{array}$ & $\begin{array}{l}88 \\
(56.8 \%)\end{array}$ & $\begin{array}{l}231 \\
(54.4 \%)\end{array}$ & $\begin{array}{l}631 \\
(54.3 \%)\end{array}$ \\
\hline Yes & $\begin{array}{l}336 \\
(45.7 \%)\end{array}$ & $\begin{array}{l}127 \\
(47.0 \%)\end{array}$ & $\begin{array}{l}67 \\
(43.2 \%)\end{array}$ & $\begin{array}{l}194 \\
(45.6 \%)\end{array}$ & $\begin{array}{l}530 \\
(45.7 \%)\end{array}$ \\
\hline \multicolumn{6}{|c|}{ Respiratory disease } \\
\hline Unknown & 3 & 0 & 0 & 0 & 3 \\
\hline No & $\begin{array}{l}605 \\
(82.4 \%)\end{array}$ & $\begin{array}{l}229 \\
(84.8 \%)\end{array}$ & $\begin{array}{l}134 \\
(86.5 \%)\end{array}$ & $\begin{array}{l}363 \\
(85.4 \%)\end{array}$ & $\begin{array}{l}968 \\
(83.5 \%)\end{array}$ \\
\hline Yes & $\begin{array}{l}129 \\
(17.6 \%)\end{array}$ & $\begin{array}{l}41 \\
(15.2 \%)\end{array}$ & $\begin{array}{l}21 \\
(13.5 \%)\end{array}$ & $\begin{array}{l}62 \\
(14.6 \%)\end{array}$ & $\begin{array}{l}191 \\
(16.5 \%)\end{array}$ \\
\hline
\end{tabular}

Diabete (Insulin-dependent type 1 diabetes and type 2 diabete)

Unknown

No

Yes

Inflammatory and/or autoimmune diseases receiving immunosuppressive treatment

Unknown

No

Yes

Immunodeficiency

Unknown

No

Yes

Organ deficiency (hepatic, renal...)

$$
\text { Unknown }
$$

No

Yes

Organ and/or stem cell transplants

Unknown

No

Yes

Smoker status

Missing

Unknown

No smoker

Current smoker

Weaning

$\begin{array}{lllll}3 & 1 & 1 & 2 & 5 \\ 627 & 224 & 130 & 354 & 981 \\ (85.4 \%) & (83.3 \%) & (84.4 \%) & (83.7 \%) & (84.8 \%) \\ 107 & 45 & 24 & 69 & 176 \\ (14.6 \%) & (16.7 \%) & (15.6 \%) & (16.3 \%) & (15.2 \%)\end{array}$

$\begin{array}{lllll}4 & 2 & 1 & 3 & 7\end{array}$

$\begin{array}{lllll}715 & 259 & 149 & 408 & 1123\end{array}$

$\begin{array}{lllll}(97.5 \%) & (96.6 \%) & (96.8 \%) & (96.7 \%) & (97.2 \%)\end{array}$

$\begin{array}{lllll}18 & 9 & 5 & 14 & 32\end{array}$

$\begin{array}{llll}(2.5 \%) & (3.4 \%) & (3.2 \%) & (3.3 \%)\end{array}$

$\begin{array}{llllll}2 & 2 & 0 & 2 & 4\end{array}$

$\begin{array}{lllll}717 & 258 & 148 & 406 & 1123\end{array}$

$\begin{array}{llll}(97.6 \%) & (96.3 \%) & (95.5 \%) & (96.0 \%)\end{array}$

$\begin{array}{lllll}18 & 10 & 7 & 17 & 35\end{array}$

$\begin{array}{llll}(2.4 \%) & (3.7 \%) & (4.5 \%) & (4.0 \%)\end{array}$

$\begin{array}{llll}0 & 3 & 1 & 4\end{array}$

$\begin{array}{lllll}611 & 233 & 139 & 372 & 983\end{array}$

$\begin{array}{lllll}(82.9 \%) & (87.3 \%) & (90.3 \%) & (88.4 \%) & (84.9 \%)\end{array}$

$\begin{array}{lllll}126 & 34 & 15 & 49 & 175\end{array}$

$\begin{array}{lllll}(17.1 \%) & (12.7 \%) & (9.7 \%) & (11.6 \%) & (15.1 \%)\end{array}$

$\begin{array}{lllll}1 & 2 & 2 & 4 & 5\end{array}$

$\begin{array}{lllll}727 & 262 & 152 & 414 & 1141\end{array}$

$\begin{array}{lllll}(98.8 \%) & (97.8 \%) & (99.3 \%) & (98.3 \%) & (98.6 \%)\end{array}$

$\begin{array}{lllll}9 & 6 & 1 & 7 & 16\end{array}$

$\begin{array}{llll}(1.2 \%) & (2.2 \%) & (0.7 \%) & (1.7 \%)\end{array}$

$\begin{array}{lllll}2 & 0 & 4 & 4 & 6 \\ 79 & 31 & 21 & 52 & 131 \\ 320 & 148 & 68 & 216 & 536 \\ (48.8 \%) & (61.9 \%) & (52.3 \%) & (58.5 \%) & (52.3 \%) \\ 124 & 33 & 20 & 53 & 177 \\ (18.9 \%) & (13.8 \%) & (15.4 \%) & (14.4 \%) & (17.3 \%) \\ 212 & 58 & 42 & 100 & 312 \\ (32.3 \%) & (24.3 \%) & (32.3 \%) & (27.1 \%) & (30.4 \%)\end{array}$

Chi-2 $P=0.032$

Chi-2 $P=0.013$

Chi-2 $P=0.750$

Chi-2 $P=0.380$

Chi-2 $P=0.696$

Chi-2 $P=0.690$

Chi-2 $P=0.292$

Chi-2 $P=0.337$ 


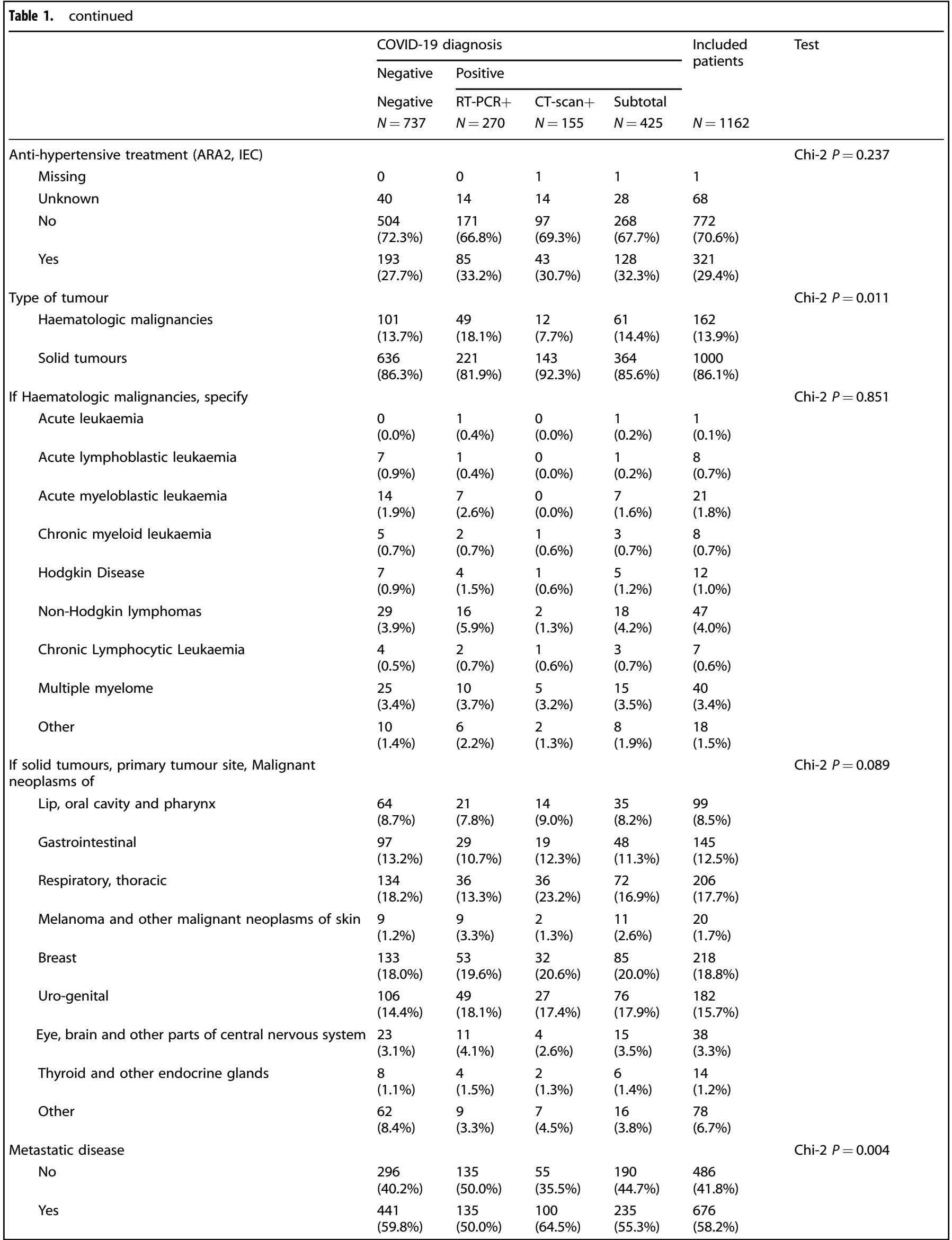


Mortality of patients with solid and haematological cancers presenting...

$S$ Assaad et al.

Table 1. continued

\begin{tabular}{|c|c|c|c|c|c|c|}
\hline & \multicolumn{4}{|c|}{ COVID-19 diagnosis } & \multirow{3}{*}{$\begin{array}{l}\text { Included } \\
\text { patients } \\
N=1162\end{array}$} & \multirow[t]{3}{*}{ Test } \\
\hline & \multirow{2}{*}{$\begin{array}{l}\text { Negative } \\
\text { Negative } \\
N=737\end{array}$} & \multicolumn{3}{|l|}{ Positive } & & \\
\hline & & $\begin{array}{l}\text { RT-PCR }+ \\
N=270\end{array}$ & $\begin{array}{l}\text { CT-scan }+ \\
N=155\end{array}$ & $\begin{array}{l}\text { Subtotal } \\
N=425\end{array}$ & & \\
\hline Presence of pulmonary metastasis & & & & & & Chi-2 $P=0.047$ \\
\hline No & $\begin{array}{l}536 \\
(72.7 \%)\end{array}$ & $\begin{array}{l}217 \\
(80.4 \%)\end{array}$ & $\begin{array}{l}115 \\
(74.2 \%)\end{array}$ & $\begin{array}{l}332 \\
(78.1 \%)\end{array}$ & $\begin{array}{l}868 \\
(74.7 \%)\end{array}$ & \\
\hline Yes & $\begin{array}{l}201 \\
(27.3 \%)\end{array}$ & $\begin{array}{l}53 \\
(19.6 \%)\end{array}$ & $\begin{array}{l}40 \\
(25.8 \%)\end{array}$ & $\begin{array}{l}93 \\
(21.9 \%)\end{array}$ & $\begin{array}{l}294 \\
(25.3 \%)\end{array}$ & \\
\hline $\begin{array}{l}\text { Time from the date of diagnosis to the date of COVID } \\
\text { test (months) }\end{array}$ & & & & & & $\begin{array}{l}\text { T-test } P=0.936 \\
\text { Kruskal-Wallis } P= \\
0.215\end{array}$ \\
\hline $\mathrm{N}$ & 736 & 268 & 155 & 423 & 1159 & \\
\hline Median (Q1-Q3) & $\begin{array}{l}12.1 \\
(4.5 ; 42.2)\end{array}$ & $\begin{array}{l}11.5 \\
(4.0 ; 37.0)\end{array}$ & $\begin{array}{l}18.8 \\
(5.4 ; 44.5)\end{array}$ & $\begin{array}{l}13.4 \\
(4.3 ; 40.9)\end{array}$ & $\begin{array}{l}12.8 \\
(4.4 ; 41.8)\end{array}$ & \\
\hline Chemotherapy & & & & & & Chi-2 $P=0.253$ \\
\hline No & $\begin{array}{l}287 \\
(38.9 \%)\end{array}$ & $\begin{array}{l}104 \\
(38.5 \%)\end{array}$ & $\begin{array}{l}71 \\
(45.8 \%)\end{array}$ & $\begin{array}{l}175 \\
(41.2 \%)\end{array}$ & $\begin{array}{l}462 \\
(39.8 \%)\end{array}$ & \\
\hline Yes & $\begin{array}{l}450 \\
(61.1 \%)\end{array}$ & $\begin{array}{l}166 \\
(61.5 \%)\end{array}$ & $\begin{array}{l}84 \\
(54.2 \%)\end{array}$ & $\begin{array}{l}250 \\
(58.8 \%)\end{array}$ & $\begin{array}{l}700 \\
(60.2 \%)\end{array}$ & \\
\hline \multicolumn{7}{|l|}{ Class of chemotherapy } \\
\hline Alkylating agents & $\begin{array}{l}40 \\
(5.4 \%)\end{array}$ & $\begin{array}{l}24 \\
(8.9 \%)\end{array}$ & $\begin{array}{l}13 \\
(8.4 \%)\end{array}$ & $\begin{array}{l}37 \\
(8.7 \%)\end{array}$ & $\begin{array}{l}77 \\
(6.6 \%)\end{array}$ & \\
\hline Antimetabolites & $\begin{array}{l}51 \\
(6.9 \%)\end{array}$ & $\begin{array}{l}22 \\
(8.1 \%)\end{array}$ & $\begin{array}{l}12 \\
(7.7 \%)\end{array}$ & $\begin{array}{l}34 \\
(8.0 \%)\end{array}$ & $\begin{array}{l}85 \\
(7.3 \%)\end{array}$ & \\
\hline Anthracyclines & $\begin{array}{l}18 \\
(2.4 \%)\end{array}$ & $\begin{array}{l}5 \\
(1.9 \%)\end{array}$ & $\begin{array}{l}2 \\
(1.3 \%)\end{array}$ & $\begin{array}{l}7 \\
(1.6 \%)\end{array}$ & $\begin{array}{l}25 \\
(2.2 \%)\end{array}$ & \\
\hline Topoisomerases I/II inhibitors & $\begin{array}{l}6 \\
(0.8 \%)\end{array}$ & $\begin{array}{l}3 \\
(1.1 \%)\end{array}$ & $\begin{array}{l}2 \\
(1.3 \%)\end{array}$ & $\begin{array}{l}5 \\
(1.2 \%)\end{array}$ & $\begin{array}{l}11 \\
(0.9 \%)\end{array}$ & \\
\hline Taxanes & $\begin{array}{l}43 \\
(5.8 \%)\end{array}$ & $\begin{array}{l}18 \\
(6.7 \%)\end{array}$ & $\begin{array}{l}14 \\
(9.0 \%)\end{array}$ & $\begin{array}{l}32 \\
(7.5 \%)\end{array}$ & $\begin{array}{l}75 \\
(6.5 \%)\end{array}$ & \\
\hline Mitotic inhibitors & $\begin{array}{l}16 \\
(2.2 \%)\end{array}$ & $\begin{array}{l}5 \\
(1.9 \%)\end{array}$ & $\begin{array}{l}6 \\
(3.9 \%)\end{array}$ & $\begin{array}{l}11 \\
(2.6 \%)\end{array}$ & $\begin{array}{l}27 \\
(2.3 \%)\end{array}$ & \\
\hline Combination & $\begin{array}{l}220 \\
(29.9 \%)\end{array}$ & $\begin{array}{l}75 \\
(27.8 \%)\end{array}$ & $\begin{array}{l}28 \\
(18.1 \%)\end{array}$ & $\begin{array}{l}103 \\
(24.2 \%)\end{array}$ & $\begin{array}{l}323 \\
(27.8 \%)\end{array}$ & \\
\hline Other & $\begin{array}{l}56 \\
(7.6 \%)\end{array}$ & $\begin{array}{l}14 \\
(5.2 \%)\end{array}$ & $\begin{array}{l}7 \\
(4.5 \%)\end{array}$ & $\begin{array}{l}21 \\
(4.9 \%)\end{array}$ & $\begin{array}{l}77 \\
(6.6 \%)\end{array}$ & \\
\hline Targeted therapy & & & & & $\begin{array}{l}\text { Chi-2 P = } \\
0.883\end{array}$ & \\
\hline No & $\begin{array}{l}613 \\
(83.2 \%)\end{array}$ & $\begin{array}{l}221 \\
(81.9 \%)\end{array}$ & $\begin{array}{l}128 \\
(82.6 \%)\end{array}$ & $\begin{array}{l}349 \\
(82.1 \%)\end{array}$ & $\begin{array}{l}962 \\
(82.8 \%)\end{array}$ & \\
\hline Yes & $\begin{array}{l}124 \\
(16.8 \%)\end{array}$ & $\begin{array}{l}49 \\
(18.1 \%)\end{array}$ & $\begin{array}{l}27 \\
(17.4 \%)\end{array}$ & $\begin{array}{l}76 \\
(17.9 \%)\end{array}$ & $\begin{array}{l}200 \\
(17.2 \%)\end{array}$ & \\
\hline \multicolumn{7}{|l|}{ Class of targeted therapy } \\
\hline Anti HER/ERBB & $\begin{array}{l}25 \\
(3.4 \%)\end{array}$ & $\begin{array}{l}9 \\
(3.3 \%)\end{array}$ & $\begin{array}{l}6 \\
(3.9 \%)\end{array}$ & $\begin{array}{l}15 \\
(3.5 \%)\end{array}$ & $\begin{array}{l}40 \\
(3.4 \%)\end{array}$ & \\
\hline Anti-angiogenic & $\begin{array}{l}27 \\
(3.7 \%)\end{array}$ & $\begin{array}{l}14 \\
(5.2 \%)\end{array}$ & $\begin{array}{l}6 \\
(3.9 \%)\end{array}$ & $\begin{array}{l}20 \\
(4.7 \%)\end{array}$ & $\begin{array}{l}47 \\
(4.0 \%)\end{array}$ & \\
\hline PI3K-AKT-mTOR Inhibitors & $\begin{array}{l}5 \\
(0.7 \%)\end{array}$ & $\begin{array}{l}2 \\
(0.7 \%)\end{array}$ & $\begin{array}{l}3 \\
(1.9 \%)\end{array}$ & $\begin{array}{l}5 \\
(1.2 \%)\end{array}$ & $\begin{array}{l}10 \\
(0.9 \%)\end{array}$ & \\
\hline RAS-RAF-MEK-ERK Inhibitors & $\begin{array}{l}5 \\
(0.7 \%)\end{array}$ & $\begin{array}{l}2 \\
(0.7 \%)\end{array}$ & $\begin{array}{l}0 \\
(0.0 \%)\end{array}$ & $\begin{array}{l}2 \\
(0.5 \%)\end{array}$ & $\begin{array}{l}7 \\
(0.6 \%)\end{array}$ & \\
\hline BCR-ABL, KIT Inhibitors & $\begin{array}{l}12 \\
(1.6 \%)\end{array}$ & $\begin{array}{l}5 \\
(1.9 \%)\end{array}$ & $\begin{array}{l}3 \\
(1.9 \%)\end{array}$ & $\begin{array}{l}8 \\
(1.9 \%)\end{array}$ & $\begin{array}{l}20 \\
(1.7 \%)\end{array}$ & \\
\hline MET Inhibitors & $\begin{array}{l}2 \\
(0.3 \%)\end{array}$ & $\begin{array}{l}0 \\
(0.0 \%)\end{array}$ & $\begin{array}{l}0 \\
(0.0 \%)\end{array}$ & $\begin{array}{l}0 \\
(0.0 \%)\end{array}$ & $\begin{array}{l}2 \\
(0.2 \%)\end{array}$ & \\
\hline Other & $\begin{array}{l}48 \\
(6.5 \%)\end{array}$ & $\begin{array}{l}17 \\
(6.3 \%)\end{array}$ & $\begin{array}{l}9 \\
(5.8 \%)\end{array}$ & $\begin{array}{l}26 \\
(6.1 \%)\end{array}$ & $\begin{array}{l}74 \\
(6.4 \%)\end{array}$ & \\
\hline
\end{tabular}




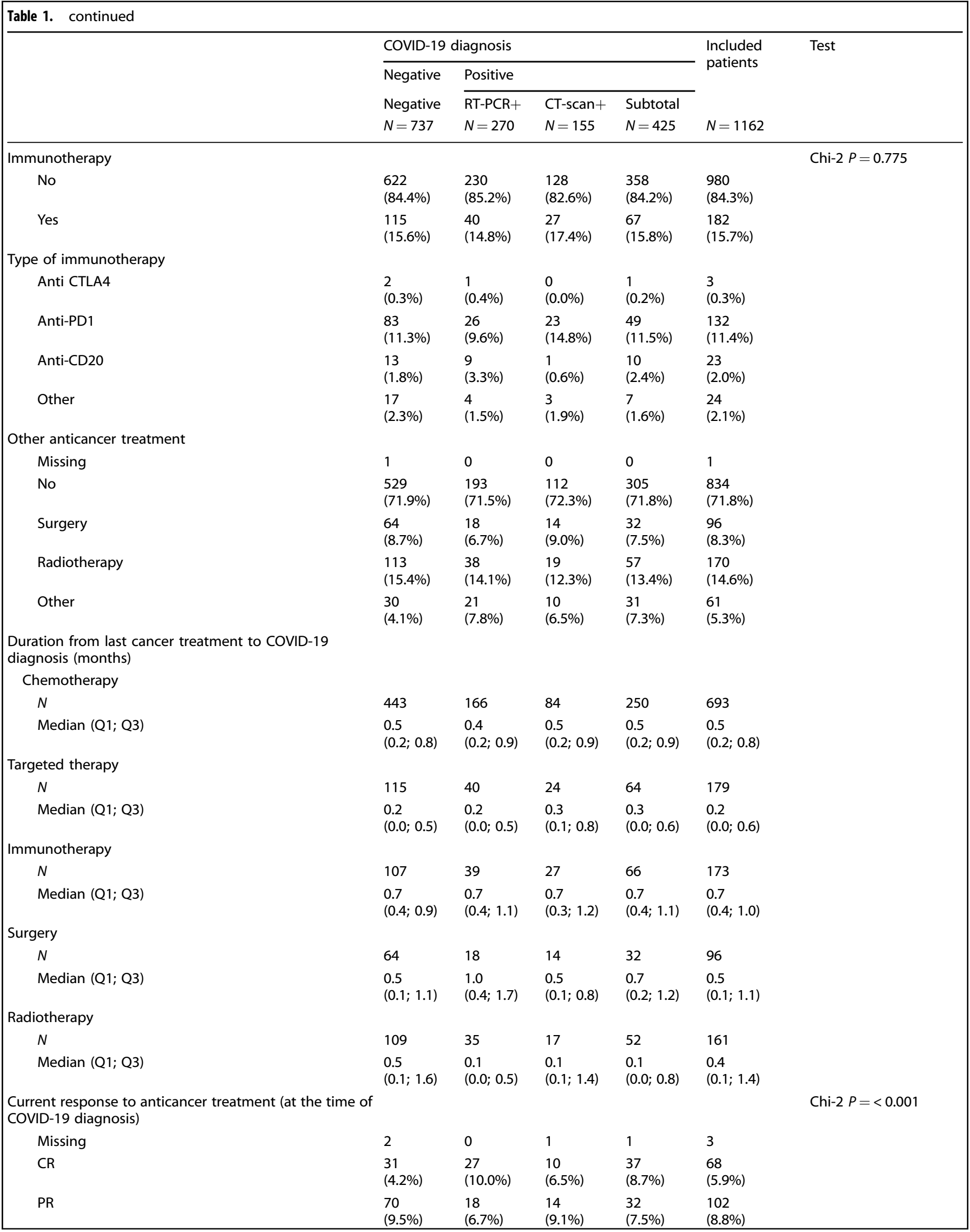




\begin{tabular}{|c|c|c|c|c|c|c|}
\hline & \multicolumn{4}{|c|}{ COVID-19 diagnosis } & \multirow{3}{*}{$\begin{array}{l}\text { Included } \\
\text { patients } \\
N=1162\end{array}$} & \multirow[t]{3}{*}{ Test } \\
\hline & \multirow{2}{*}{$\begin{array}{l}\text { Negative } \\
\text { Negative } \\
N=737\end{array}$} & \multicolumn{3}{|l|}{ Positive } & & \\
\hline & & $\begin{array}{l}\text { RT-PCR }+ \\
N=270\end{array}$ & $\begin{array}{l}\text { CT-scan+ } \\
N=155\end{array}$ & $\begin{array}{l}\text { Subtotal } \\
N=425\end{array}$ & & \\
\hline SD & $\begin{array}{l}118 \\
(16.1 \%)\end{array}$ & $\begin{array}{l}34 \\
(12.6 \%)\end{array}$ & $\begin{array}{l}19 \\
(12.3 \%)\end{array}$ & $\begin{array}{l}53 \\
(12.5 \%)\end{array}$ & $\begin{array}{l}171 \\
(14.8 \%)\end{array}$ & \\
\hline $\mathrm{NE}$ & $\begin{array}{l}349 \\
(47.5 \%)\end{array}$ & $\begin{array}{l}153 \\
(56.7 \%)\end{array}$ & $\begin{array}{l}70 \\
(45.5 \%)\end{array}$ & $\begin{array}{l}223 \\
(52.6 \%)\end{array}$ & $\begin{array}{l}572 \\
(49.4 \%)\end{array}$ & \\
\hline Continuation of anticancer treatment during the study & & & & & & Chi-2 $P=0.002$ \\
\hline Missing & 1 & 0 & 0 & 0 & 1 & \\
\hline Corticotherapy as anticancer treatment & & & & & & Chi-2 $P=0.390$ \\
\hline No & $\begin{array}{l}570 \\
(77.3 \%)\end{array}$ & $\begin{array}{l}219 \\
(81.1 \%)\end{array}$ & $\begin{array}{l}124 \\
(80.0 \%)\end{array}$ & $\begin{array}{l}343 \\
(80.7 \%)\end{array}$ & $\begin{array}{l}913 \\
(78.6 \%)\end{array}$ & \\
\hline Yes & $\begin{array}{l}167 \\
(22.7 \%)\end{array}$ & $\begin{array}{l}51 \\
(18.9 \%)\end{array}$ & $\begin{array}{l}31 \\
(20.0 \%)\end{array}$ & $\begin{array}{l}82 \\
(19.3 \%)\end{array}$ & $\begin{array}{l}249 \\
(21.4 \%)\end{array}$ & \\
\hline
\end{tabular}

$B M I$ body mass index, ECOG-PS Eastern Cooperative Oncology Group performance status, ECMO extracorporal membrane oxygenation, ARA2 angiotensin receptor 2, IEC angiotensin converting enzyme, $K W$ Kruskal-Wallis.

Data are expressed $n(\%)$, and median (Q1-Q3).

oxygen $(N=179,53 \%)$, glucocorticoid therapy $(N=47,13.9 \%)$ and non-invasive ventilation $(N=40,11.9 \%)$.

\section{Survival}

In total, 234 (20.5\%) deaths within 28 days occurred in the global population $(N=1162)$, including $116 / 425(27.8 \%)$ in COVID-19positive patients and 118/737 (16.3\%) in COVID-19-negative patients (Fig. 2a $p<0.0001$ ) (see also Supplementary Table S3). With a median follow-up of 2.1 (1.6-2.4) months, $310(26.7 \%)$ deaths were reported, including 143 (33.6\%) deaths in the COVID19-positive population. According to investigators, 73 (51\%) COVID-19-specific death occurred in the COVID-19-positive group; other primary causes of death were cancer $(N=50,34.9 \%)$, and other causes $(20,13.9 \%)$. Investigators reported the cause of deaths, and in the case of COVID-19-specific death, this information was documented on death certificate. In the 737 COVID-19-negative patient population, 167 (22.7\%) deaths occurred: the physicians in charge reported deaths as related to cancer $(N=138,82.6 \%)$, while $29(17.4 \%)$ deaths were attributed to other causes.

Figure 2 shows the differential survival between the COVID-19negative patients and -positive patients as a whole (Fig. $2 \mathrm{a}, p<$ 0.001 ), and in 3 subgroups (Fig. 2B) COVID-19 patients (i) positive according to RT-PCR, (ii) positive according to $C T$ scan and (iii) negative for COVID-19 RT-PCR results with inconclusive chest X-ray or CT scans. The two COVID-19-positive subgroups showed no statistically significant differences in survival $(p=0.1508)$, though COVID-19-positive patients identified by chest CT-scan only (without detectable SARS-COV-2 on RT-PCR) had graphically a worse survival.

Predictive factors for death

Predictive factors for death within 28 days in the global population and in the COVID-19-positive population (RT-PCR+ and/or CT-scan+) were investigated (Supplementary Table S4).
Baseline CRP levels were missing in $N=269$ (23.0\%) patients. The median baseline CRP level in the global population was 70.2 (25-145) $\mathrm{mg} / \mathrm{L}, 57.3(20-122) \mathrm{mg} / \mathrm{L}$ in patients alive at day 28 , vs $135.3 \mathrm{mg} / \mathrm{dL}$ (71-219) in patients who died before day 28. Among the 893 patients with CRP measured at baseline, death at day 28 occurred in 50 of the $446(11.4 \%)$ patients with $\leq 70 \mathrm{mg} / \mathrm{L}$ baseline CRP, and 150/447 (33.7\%) with $>70 \mathrm{mg} / \mathrm{L}$ baseline CRP $(p<0.001)$, and in $24 / 159(15.3 \%)$ and $76 / 166(46.1 \%)$ in the COVID-19positive subgroup $(p<0.001)$ (Fig. 3). A baseline CRP $>70 \mathrm{mg} / \mathrm{L}$ was found associated with a major increase of the risk of death at day 28 for cancer patients with COVID-19. However, the $23 \%$ rate of missing values prevented this variable to be included in the multivariate model.

Multivariate analyses show that male gender, metastatic disease, history of inflammatory or autoimmune disease receiving immunosuppressive treatments, lymphopenia $(\leq 0.7 \mathrm{G} / \mathrm{L})$, diabetes and SARS-COV-2 positivity (see details in the table) were significantly associated with increased mortality in the global population (Table 2A).

In the COVID-19-positive patient population, male gender, metastatic disease, history of inflammatory or autoimmune disease receiving immunosuppressive treatments and lymphopenia $\leq 0.7 \mathrm{G} / \mathrm{L}$ were predictive factors for death (Table $2 \mathrm{~B}$ ).

Lymphopenia $\leq 0.7 \mathrm{G} / \mathrm{L}$ and metastatic status were independent risk factors of death in the global population and in the COVID-19positive population (Table 2). Survival curves according to lymphocyte rate and metastatic status in the COVID-19-positive and COVID-19-negative population are presented in Supplementary Fig. S1.

None of the histotypes of cancer and none of the classes of anticancer treatment (cytotoxics, anti-CD20, targeted therapy excluding antiangiogenics, PD1 and other checkpoint inhibitors) were significantly associated with an increased risk of death in univariate or multivariate analysis in the global population and in the COVID-19-positive population (Table 2, Supplementary Figs. S1-S3). 
Table 2. Multivariate analysis of risk factors for death at day 28 in (A) global population and (B) in COVID-19 positive patients.

Odds ratio estimates

Effect

Point estimate

$95 \%$ Wald

confidence limits

(A) Global population

Gender

1.836

1.328

2.539

0.0002

Male vs Female

History of diabetes

1.645

1.083

2.500

Yes vs No

History of inflammatory and/or autoimmune diseases receiving immunosuppressive treatment Yes vs No

Metastatic disease

3.205

1.392

7.376

0.0062

Yes vs No

Lymphocytes (baseline)

2.844

1.975

4.097

$<0.0001$

$\leq 0.7 \mathrm{G} / \mathrm{L}$ vs $>0.7 \mathrm{G} / \mathrm{L}$

1.813

1.315

2.501

0.0003

COVID-19 diagnosis result

COVID-19 Positive diagnosis (RT-PCR+) vs Negative diagnosis

COVID-19 Positive diagnosis (CT-scan+) vs Negative diagnosis

2.192

1.5052

3.194

(B) COVID-19 positive population

Gender

1.358

3.285

Male vs Female

History of Inflammatory and/or autoimmune diseases receiving immunosuppressive treatment

1.477

3.893

0.0004

Yes vs No

Metastatic disease

4.441

15.545

0.0197

Yes vs No

3.232

1.898

5.505

$<0.0001$

Lymphocytes

1.970

1.204

3.222

0.0070

$\leq 0.7 \mathrm{G} / \mathrm{L}$ vs $>0.7 \mathrm{G} / \mathrm{L}$

(A) Gender, age, history of cardiovascular disease, history of diabetes, history of autoimmune/ inflammatory disease related to cancer, time from cancer diagnosis to the date of COVID-19 diagnosis, type of tumour, metastatic disease, pulmonary metastases, lymphopenia, time from the date of surgery to the date of the test, overall test result ( 3 modalities) have been included as variables.

(B) Gender, age, history of diabetes, history of autoimmune/inflammatory disease, smoker status, time from the date of diagnosis to the date of COVID-19 test, type of tumour, metastatic disease, pulmonary metastases, lymphopenia, ongoing corticotherapy for cancer have been included as variables.

\section{DISCUSSION}

The objective of the present nationwide multicentric study was to investigate the clinical characteristics of patients with solid tumours or haematological malignancies presenting COVID-19 symptoms, the impact of a confirmed COVID-19 diagnosis on survival, and to explore predictive factors for survival, including specific cancer treatments.

Only $36 \%$ of cancer patients presenting with COVID-19 symptoms had documented COVID-19 infection. The COVID-19 population was identified using RT-PCR and/or typical radiological signs on chest CT scan. Importantly bronchioalveolar aspiration for bacteriologic, fungal and virologic infection testing had been performed in COVID-19-positive patients (CT-scan+, RT-PCR-) in order to exclude any other possible causes of infections. ${ }^{23,27}$

In practice, 270 (63.5\%) of the COVID-19-positive patients are identified by nasopharyngeal SARS-COV-2 RT-PCR. Among the symptomatic cancer patients tested twice and identified as negative for SARS-COV-2 by RT-PCR, 155 (36\%) patients were classified as COVID-19 positive based on COVID-19 typical CT scans, a proportion in line with other published studies. ${ }^{25}$ This series shows a significant proportion of cancer patients likely infected by SARS-COV-2 who remain negative by RT-PCR, but specific CT scans. The clinical presentation and treatment characteristics of COVID-19-positive (RT-PCR+ or RT-PCR-/CTscan+) and -negative cancer patient populations were not significantly different. Our series identified no significant differences in survival among COVID-19- positive patients (RT-PCR+ or
RT-PCR-/CT-scan+). However, the present study showed a significant difference in mortality within one month after COVID19 diagnosis, with 116 (27.8\%) deaths in COVID-19-positive patient population, compared to $118(16.3 \%)$ deaths in patients categorised as COVID-19 negative. These results are consistent with previous reports showing that cancer patients are at high risk of death after documented COVID-19. ${ }^{5-18}$ This $28 \%$ death rate is in the upper range of previous reports, confirming that cancer patients in therapeutic phase are a population at high risk for death. This present series identified COVID-19 as the main cause of death for $50 \%$ of the patients with solid tumours or haematological malignancies, according to the investigators.

The present study also shows a high mortality rate in cancer patients presenting with COVID-19 symptoms, but without detectable SARS-COV-2 by RT-PCR nor documented CT scan. With $16 \%$ death rate at day 28 , the death rate is significantly lower in this group than in patients with documented SARS-COV-2 RT-PCR + ; however, this death rate at day 28 is higher than standard population of cancer patients. ${ }^{28,29}$ The current results confirm observations reported in our previous single-centre study. ${ }^{18}$ In this patient population, death is considered to be mostly $(>50 \%)$ related to the underlying malignancy as per investigators' assessment. We would nevertheless be cautious in the absence of other documentation for infection.

This mortality rate is higher than previously reported in a similar population of cancer patients outside of the present epidemic context. $^{28,30,31}$ This suggests that a significant proportion of 
COVID-19 negative vs COVID-19 positive ( ${ }^{*} \mathrm{RT}-\mathrm{PCR}+\mathrm{and}{ }^{* \star} \mathrm{CT}$-scan+) subgroups

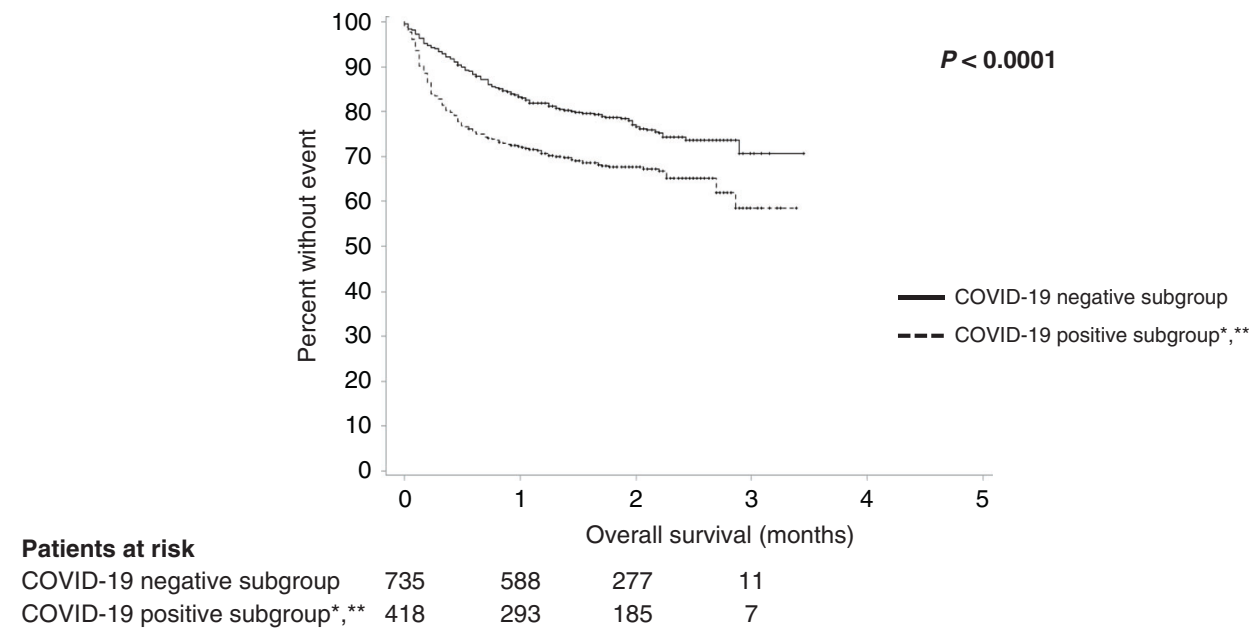

b

COVID-19 negative vs COVID-19 positive ( ${ }^{*} \mathrm{RT}-\mathrm{PCR}+$, or $\left.{ }^{*} \mathrm{CT}-\mathrm{scan}+\right)$ subgroups

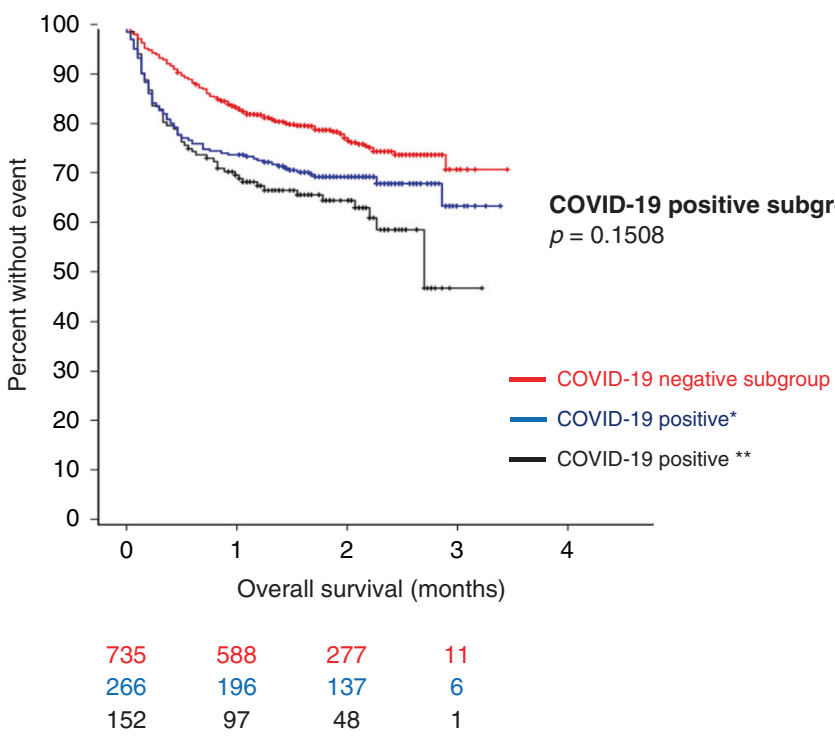

Fig. 2 Overall survival in the global population and according to COVID-19 status. a COVID-19-negative versus COVID-19-positive ( ${ }^{*} \mathrm{RT}-\mathrm{PCR}$ + and ${ }^{* *} \mathrm{CT}$-scan +$)$ subgroups, b COVID-19-negative versus COVID-19-positive ( $\left.{ }^{*} \mathrm{RT}-\mathrm{PCR}+\right)$ and COVID-19-positive $\left({ }^{* *} \mathrm{CT}\right.$-scan +$)$ subgroups.

patients had false-negative tests, despite double testing 7 days apart, and that some of these patients failed to be diagnosed as positive though being actually infected by SARS-COV-2 and undiagnosed yet. False-negative rate of COVID-19 diagnostic tests, both for RT-PCR ${ }^{22}$ and serodiagnostics, ${ }^{23}$ varied considerably, in particular in cancer patients. ${ }^{24} \mathrm{~A}$ non-mutually exclusive hypothesis is that deaths are related to other undiagnosed infection. $\mathrm{H} 1 \mathrm{~N} 1$ mortality rates were about $0.3 \%$ to $1.5 \%$ i.e. $3-10$ times higher than seasonal influenza in the general population. The mortality rates in cancer patients ranged from $9 \%$ after seasonal influenza, and $9 \%$ to $25 \%$ following H1N1 outbreak showing that cancer patients with severe symptoms of viral infection are generally at high risk for death from respiratory tract viral infections. ${ }^{19-21}$ In comparison, while cancer has likely contributed to death rate of these patients, this high death rate observed here and in a previous work ${ }^{18}$ suggests a possible underestimation of the influence of COVID-19 in the lethality of these infectious events.

The representativity of the patient population tested here must be commented. This research was proposed early in the epidemic, and access was provided to a representative variety of French cancer patients through the rapid involvement of 23 institutions, Comprehensive Cancer Centers as well as general hospitals. At that date, the national testing policy mostly used RT-PCR in clinically severe cases, possibly accounting for the overall poor prognosis of cancer patients. Even though all authorised sites included all cancer patients with COVID-19 suspicion, we cannot exclude an under-reporting of COVID-19 in cancer patients with all types of malignancies, particularly in patients with mild symptoms or in asymptomatic patients who did not attend medical services. Moreover, not all cancer patients attending participating centres have been tested for COVID-19, and our patient population probably selected cancer patients with more severe form of the infection.

Several risk factors for early death were identified. Independent risk factors for death were gender, metastases, history of inflammatory and/or autoimmune disease and lymphopenia at the time of suspected COVID-19, which is consistent with the literature. ${ }^{13,14,16-18}$ However, the present series did not identify age, BMI, cardiovascular risk factors or the nature of the underlying malignancy (solid tumour or haematological) as independent risk factors, possibly because of the limited 


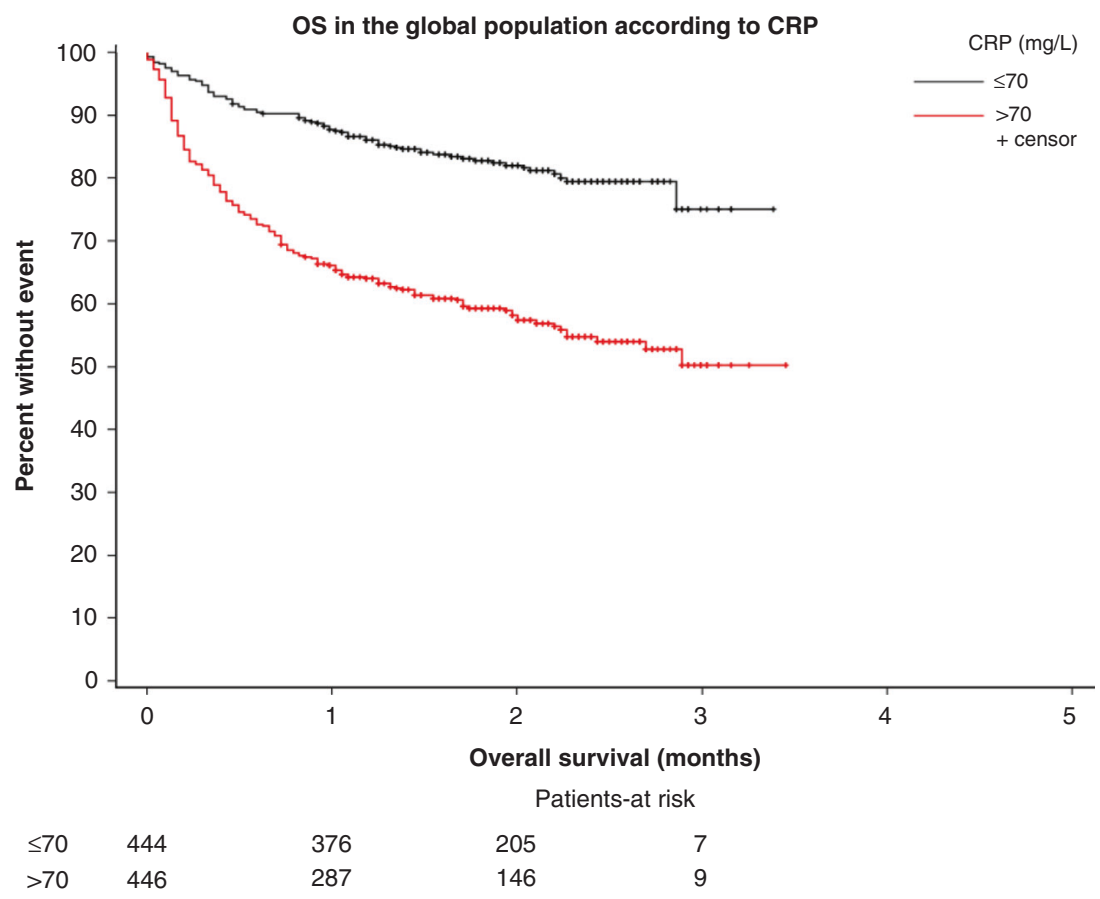

b

COVID-19 positive patients

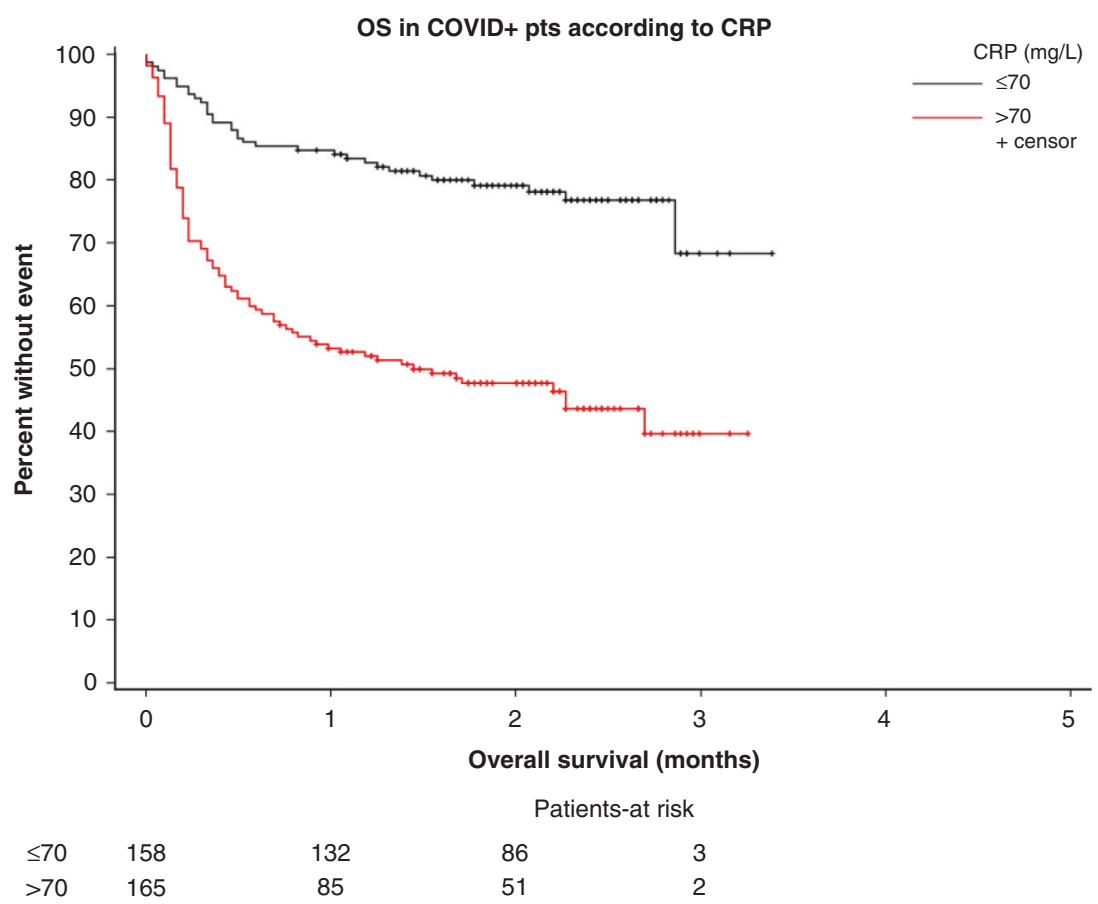

Fig. 3 Survival of the global and COVID-19-positive groups according to baseline CRP levels ( $\leq 70 \mathrm{mg} / \mathrm{L},>70 \mathrm{mg} / \mathrm{L}$ ). a Global population, b COVID-19-positive population.

proportion of $\mathrm{BMI}>30$ and the death rate due to cancer in the younger group of patients. Lymphopenia has been previously reported as a major risk factor for early death in cancer patient population. ${ }^{28,31}$ CRP levels were documented in only $77 \%$ of patients precluding integration in a multivariate analysis. However, high CRP levels were associated with a 34\% death rate at day 28 for all patients presenting with a CRP $>70 \mathrm{mg} / \mathrm{L}$, and up to $46 \%$ for COVID-19-positive patients, which therefore constitutes a population at very high risk for death. Serum CRP increase is induced by IL-6, also known as a poor prognostic factor for survival either in patients with COVID-19, ${ }^{32}$ or in patients with cancer. ${ }^{27}$ CRP levels may be easily used as a simple biological parameter to identify patients with a dismal prognosis and further exploration in this patient population is required. 
Importantly, the safety of anticancer treatments has been discussed early in the COVID-19 pandemic, and potential risks associated with chemotherapy, targeted therapy and immune checkpoint inhibitors have been questioned. In France, cancer patients were treated as recommended by national and international guidelines. ${ }^{33,34}$ In practice, cancer patients treated with chemotherapy or targeted therapy received the regular recommended scheme, thoracic radiotherapy with curative intent was continued, oncologic surgeries and more specifically thoracic oncology surgeries were maintained in the prescheduled timelines with adapted protocols. Whether the use of immune checkpoint inhibitors in cancer patients may enhance the cytokine storm associated with COVID-19 has emerged, and decisionmaking to treat immune-mediated toxicities with immunesuppressive steroids was debated. ${ }^{35}$ Guidelines in the United Kingdom have suggested to proceed with caution or avoid/ interrupt treatments. ${ }^{36}$ In France, we locally implemented adapted therapeutic administration schemes for immune checkpoint inhibitors while global dose intensity was respected. More specifically, patients with lung cancer received immune checkpoint inhibitors with extended duration between visits (nivolumab $2 w \rightarrow 4 w ;$ Pembrolizumab $3 w \rightarrow 6 w ;$ Durvalumab $2 w \rightarrow 4 w$ ) according to national recommendations in thoracic oncology, in order to minimise hospital visits for patients, thereby limiting the risks of contamination. Surveillance through regular nurse phone call and health teleconsultations was performed for early detection of potential treatment-related toxicities and complications, or any emergent COVID-19 symptoms.

In contrast with previous reports, none of the cancer treatments, including immunotherapies, anti-CD20 and tyrosine kinase inhibitors, were associated with a significantly different mortality rate in our population. ${ }^{13}$ Our results are consistent with the results from Lee et al., ${ }^{8}$ and delays in referral pathway or treatment should not be extended. 37,38

Finally, it must be noted that no COVID-19-specific treatments were given to these patients. Their effectiveness at this time was unclear. The magnitude of benefit provided by hydrochloroquine, remdesivir or anti-IL- 6 was unclear or limited on survival, even though a significant reduction of the duration in intensive care unit and hospitalisation was noted for the anti-IL-6 trial. ${ }^{39-42}$

The histological subtype or location of cancer was not correlated to death rate at day 28 either, also in contrast with other reports, probably reflecting different population selections. While a combined analysis of published data would help to achieve progress on this question, this unselected cancer patient population with either haematological malignancies or solid tumours of various histotypes and treatments suggests that the nature of cancer treatment is not a major parameter related to the death of cancer patients during this epidemic.

In conclusion, this nationwide series shows that a documented COVID-19 infection was associated with a $28 \%$ day- 28 mortality rate in cancer patients, regardless of the treatment given for the underlying cancer, and regardless of the cancer type. The death rate was $16 \%$ in patients without COVID-19-positive diagnosis by RT-PCR nor typical radiological imaging, and with no other infectious documentation suggesting a significant rate of falsenegative COVID-19 diagnosis. Patients with CRP levels above 70 $\mathrm{mg} / \mathrm{L}$ are at very high risk of death at 28 days.

Our results point to the need of a careful clinical management of cancer patients presenting with severe symptoms compatible with COVID-19 with or without a confirmed virologic diagnosis as the COVID-19 pandemic seems likely to extend for several additional months.

\section{ACKNOWLEDGEMENTS}

The authors thank all the patients who participated in the study, investigational sites' staffs, oncologists, physicians and health care workers, all local investigators who included patients and the clinical research staff, especially Caroline Hernandez, Sophie Kaiser, Jérémie Monfray, Céline Ferlay and all clinical research assistants at all the participating sites. The authors thank Sophie Darnis (Centre Léon Bérard) for medical editorial assistance with this paper.

\section{AUTHOR CONTRIBUTIONS}

S.A., P.Z., C.C., J.G., O.F., D.P. and J.Y.B. contributed to the trial conception and design. S.A., P.Z., C.F., B.G., A.L.S.L., S.L., D.V., S.P., F.C., L.S., J.Pr., T.L.B., X.D., J.Pe., R.S., G.C., M.B., B.B., A.D., M.H., D.D., P.R., C.B.P. and B.C.G. contributed to data collection. C.C. did the statistical analysis and contributed together with S.A., J.G., D.P. and J.Y.B. to data analysis and interpretation. J.G. and D.P. supervised the study. All authors reviewed the paper for intellectual content, provided comments and gave final approval for publication.

\section{ADDITIONAL INFORMATION}

Ethics approval and consent to participate Local approval from Data Protection Officer of Centre Léon Bérard was obtained in accordance with French data protection authority requirements (Commission Nationale de I'Informatique et des Libertés (CNIL) MR-004 project reference INDS_090720, R201-004-040) and French and European laws and directives. Patients received an information sheet and were offered the possibility to accept or refuse to participate in this noninterventional study.

Consent to publish Not applicable.

Data availability Data supporting the findings of this study are available within the article, in the supplementary files and from the corresponding authors upon reasonable request.

Competing interests The authors declare no competing interests.

Funding information This work was partly supported by grants of the French National Research Agency (ANR) [LabEx DEvweCAN ANR-10-LABX0061], Hospitalo Universitary Research RHU4 DEPGYN [ANR-18-RHUS-0009]; Institut National du Cancer (INCa) \& Direction Générale de l'offre de soins (DGOS) [NetSARC, RREPS, RESOS, LYRICAN] [INCA-DGOS-INSERM 12563]; INCA [InterSARC]; European commission [EURACAN-EC 739521]; Fondation ARC; PIA Institut Convergence François Rabelais PLAsCAN [PLASCAN, 17-CONV-0002]; La Ligue de L'Ain contre le Cancer; La Ligue contre le Cancer funded this study. It was also partially supported by an unrestricted grant from Astra Zeneca. Role of funding source: The funders of the study had no role in study design, data collection, data analysis, data interpretation or writing of the report. S.A., C.C., J.G., D.P. and J.Y.B. had full access to all the data of the study and had final responsibility for the decision to submit for publication.

Supplementary information The online version contains supplementary material available at https://doi.org/10.1038/s41416-021-01452-4.

Publisher's note Springer Nature remains neutral with regard to jurisdictional claims in published maps and institutional affiliations.

\section{REFERENCES}

1. Guan, W. J., Ni, Z. Y., Hu, Y., Liang, W. H., Ou, C. Q., He, J. X. et al. Clinical characteristics of coronavirus disease 2019 in China. N. Engl. J. Med 382, 1708-20 (2020).

2. World Health Organisation. Coronavirus. Situation reports-179, https://www.who.int/ docs/default-source/coronaviruse/situation-reports/20200717-covid-19-sitrep-179. pdf?sfvrsn=2f1599fa_2. Posted online 17 July 2020. (2020).

3. Centers for Disease Control and Prevention. https://www.cdc.gov/coronavirus/ 2019-ncov/need-extra-precautions/older-adults.html (2020).

4. Zhang, J. J. Y., Lee, K. S., Ang, L. W., Leo, Y. S. \& Young, B. E. Risk factors for severe disease and efficacy of treatment in patients infected with COVID-19: a systematic review, meta-analysis, and meta-regression analysis. Clin. Infect. Dis. 71, 2199-206c (2020).

5. Liang, W., Guan, W., Chen, R., Wang, W., Li, J., Xu, K. et al. Cancer patients in SARS-CoV-2 infection: a nationwide analysis in China. Lancet Oncol. 21, 335-7 (2020).

6. Wang, H. \& Zhang, L. Risk of COVID-19 for patients with cancer. Lancet Oncol. 21, e181 (2020). 
7. Zhang, L., Zhu, F., Xie, L., Wang, C., Wang, J., Chen, R. et al. Clinical characteristics of COVID-19-infected cancer patients: a retrospective case study in three hospitals within Wuhan, China. Ann. Oncol. 31, 894-901 (2020).

8. Lee, L. Y., Cazier, J. B., Angelis, V., Arnold, R., Bisht, V., Campton, N. A. et al. COVID19 mortality in patients with cancer on chemotherapy or other anticancer treatments: a prospective cohort study. Lancet 395, 1919-26 (2020).

9. Kuderer, N. M., Choueiri, T. K., Shah, D. P., Shyr, Y., Rubinstein, S. M., Rivera, D. R. et al. Clinical impact of COVID-19 on patients with cancer (CCC19): a cohort study. Lancet 395, 1907-18 (2020).

10. Mehta, V., Goel, S., Kabarriti, R., Cole, D., Goldfinger, M., Acuna-Villaorduna, A. et al. Case fatality rate of cancer patients with COVID-19 in a New York Hospital System. Cancer Disco. 10, 935-41 (2020).

11. Miyashita, H., Mikami, T., Chopra, N., Yamada, T., Chernyavsky, S., Rizk, D. et al. Do patients with cancer have a poorer prognosis of COVID-19? An experience in New York City. Ann. Oncol. https://doi.org/10.1016/j.annonc.2020.04.006 (2020).

12. Whisenant, J. G., Trama, A., Torri, V., De Toma, A., Viscardi, G., Cortellini, A. et al. TERAVOLT: Thoracic cancers international COVID-19 Collaboration. Cancer Cell 37, 742-5 (2020)

13. Garassino, M. C., Whisenant, J. G., Huang, L. C., Trama, A., Torri, V., Agustoni, F. et al. COVID-19 in patients with thoracic malignancies (TERAVOLT): first results of an international, registry-based, cohort study. Lancet Oncol. 21, 914-22 (2020).

14. Liang, W.H., Guan, W.J., Li, C.C., Li, Y.M., Liang, H.R., Zhao, Y. et al. Clinical characteristics and outcomes of hospitalised patients with COVID-19 treated in Hubei (epicentre) and outside Hubei (non-epicentre): a nationwide analysis of China. Eur. Respir. J. 55, 2000562 (2020).

15. Zhang, H., Wang, L., Chen, Y., Wu, Q., Chen, G., Shen, X. et al. Outcomes of novel coronavirus disease 2019 (COVID-19) infection in 107 patients with cancer from Wuhan, China. Cancer https://doi.org/10.1002/cncr.33042 (2020).

16. Yu, J., Ouyang, W., Chua, M. L. K. \& Xie, C. SARS-CoV-2 transmission in patients with cancer at a tertiary care hospital in Wuhan, China. JAMA Oncol. 6, 1108-1110 (2020).

17. Xia, Y., Jin, R., Zhao, J., Li, W. \& Shen, H. Risk of COVID-19 for patients with. Cancer Lancet Oncol. 21, e180 (2020).

18. Assaad, S., Avrillon, V., Fournier, M. L., Mastroianni, B., Russias, B., Swalduz, A. et al. High mortality rate in cancer patients with symptoms of COVID-19 with or without detectable SARS-COV-2 on RT-PCR. Eur. J. Cancer 135, 251-259 (2020).

19. Angeles-Sistac, D., Martin-Onraet, A., Cornejo-Juarez, P., Volkow, P., Perez-Jimenez, C. \& Vilar-Compte, D. Influenza in patients with cancer after 2009 pandemic AH1N1: An 8-year follow-up study in Mexico. Influenza Other Respir. Viruses 14, 196-203 (2020).

20. Dignani, M. C., Costantini, P., Salgueira, C., Jordan, R., Guerrini, G., Valledor, A. et al. Pandemic 2009 Influenza A (H1N1) virus infection in cancer and hematopoietic stem cell transplant recipients; a multicenter observational study. F1000Res 3, 221 (2014).

21. Cooksley, C. D., Avritscher, E. B., Bekele, B. N., Rolston, K. V., Geraci, J. M. \& Elting, L. $\mathrm{S}$. Epidemiology and outcomes of serious influenza-related infections in the cancer population. Cancer 104, 618-28 (2005).

22. Kucirka, L. M., Lauer, S. A., Laeyendecker, O., Boon, D. \& Lessler, J. Variation in false-negative rate of reverse transcriptase polymerase chain reaction-based SARS-CoV-2 tests by time since exposure. Ann. Intern Med. 173, 262-7 (2020).

23. Deeks, J.J., Dinnes, J., Takwoingi, Y., Davenport, C., Spijker, R., Taylor-Phillips, S. et al. Antibody tests for identification of current and past infection with SARSCoV-2. Cochrane Database Syst. Rev. https://doi.org/10.1002/14651858.CD013652 (2020).

24. Solodky, M. L., Galvez, C., Russias, B., Detourbet, P., N'Guyen-Bonin, V., Herr, A. L. et al. Lower detection rates of SARS-COV2 antibodies in cancer patients versus health care workers after symptomatic COVID-19. Ann. Oncol. 8, 1087-1088 (2020).
25. Korevaar, D. A., Kootte, R. S., Smits, L. P., van den Aardweg, J. G., Bonta, P. I., Schinkel, J. et al. Added value of chest computed tomography in suspected COVID-19: an analysis of 239 patients. Eur. Respir. J. 56, 2001377 (2020).

26. Falaschi, Z., Danna, P. S. C., Arioli, R., Pasche, A., Zagaria, D., Percivale, I. et al. Chest CT accuracy in diagnosing COVID-19 during the peak of the Italian epidemic: A retrospective correlation with RT-PCR testing and analysis of discordant cases. Eur. J. Radio. 130, 109192 (2020).

27. Blay, J. Y., Negrier, S., Combaret, V., Attali, S., Goillot, E., Merrouche, Y. et al. Serum level of interleukin 6 as a prognosis factor in metastatic renal cell carcinoma. Cancer Res. 52, 3317-22 (1992).

28. Ray-Coquard, I., Cropet, C., Van Glabbeke, M., Sebban, C., Le Cesne, A., Judson, I. et al. Lymphopenia as a prognostic factor for overall survival in advanced carcinomas, sarcomas, and lymphomas. Cancer Res. 69, 5383-91 (2009).

29. Lee, J., Park, S. S., Kim, T. Y., Lee, D. G. \& Kim, D. W. Lymphopenia as a biological predictor of outcomes in COVID-19 patients: a nationwide cohort study. Cancers (Basel) 13, 471 (2021).

30. Ray-Coquard, I., Ghesquiere, H., Bachelot, T., Borg, C., Biron, P., Sebban, C. et al. Identification of patients at risk for early death after conventional chemotherapy in solid tumours and lymphomas. Br. J. Cancer 85, 816-22 (2001).

31. Borg, C., Ray-Coquard, I., Philip, I., Clapisson, G., Bendriss-Vermare, N., MenetrierCaux, C. et al. CD4 lymphopenia as a risk factor for febrile neutropenia and early death after cytotoxic chemotherapy in adult patients with cancer. Cancer 101 2675-80 (2004).

32. Chen, X., Zhao, B., Qu, Y., Chen, Y., Xiong, J., Feng, Y. et al. Detectable serum SARSCoV-2 viral load (RNAaemia) is closely correlated with drastically elevated interleukin 6 (IL-6) level in critically ill COVID-19 patients. Clin. Infect. Dis. 71, 1937-1942 (2020).

33. Curigliano, G., Banerjee, S., Cervantes, A., Garassino, M.C., Garrido, P., Girard, N et al. Managing cancer patients during the COVID-19 pandemic: an ESMO multidisciplinary expert consensus. Ann. Oncol. 31, 1320-1335 (2020).

34. Slimano, F., Baudouin, A., Zerbit, J., Toulemonde-Deldicque, A., Thomas-Schoemann, A., Chevrier, R. et al. Cancer, immune suppression and Coronavirus Disease-19 (COVID-19): Need to manage drug safety (French Society for Oncology Pharmacy [SFPO] guidelines). Cancer Treat. Rev. 88, 102063 (2020).

35. Kattan, J., Kattan, C. \& Assi, T. Do checkpoint inhibitors compromise the cancer patients' immunity and increase the vulnerability to COVID-19 infection? Immunotherapy 12, 351-354 (2020).

36. NHS England interim treatment options during the COVID-19 pandemic. https:// www.nice.org.uk/guidance/ng161/resources/interim-treatment-change-optionsduring-the-covid19-pandemic-endorsed-by-nhs-england-pdf-8715724381 (2021)

37. Girard, N., Greillier, L., Zalcman, G., Cadranel, J., Moro-Sibilot, D., Mazieres, J. et al. Proposals for managing patients with thoracic malignancies during COVID-19 pandemic Respir. Med. Res. 78, 100769 (2020).

38. Sud, A., Torr, B., Jones, M. E., Broggio, J., Scott, S., Loveday, C. et al. Effect of delays in the 2-week-wait cancer referral pathway during the COVID-19 pandemic on cancer survival in the UK: a modelling study. Lancet Oncol. 21, 1035-1044 (2020).

39. Grein, J., Ohmagari, N., Shin, D., Diaz, G., Asperges, E., Castagna, A. et al. Compassionate Use of Remdesivir for Patients with Severe Covid-19. N. Engl. J. Med 382, 2327-36 (2020).

40. Beigel, J.H., Tomashek, K.M., Dodd, L.E., Mehta, A.K., Zingman, B.S., Kalil, A.C. et al. Remdesivir for the treatment of Covid-19-preliminary report. N Engl. J. Med. https://doi.org/10.1056/NEJMoa2007764 (2020).

41. Rosas, I.O., Brau, N., Waters, M., Go, R., Hunter, B.D., Baghani, S. et al. Tocilizumab in hopitalized patients with COVID-19 pneumonia. N. Engl. J. Med. 384 1503-1516 (2020).

42. Tomazini, B.M., Maia, I.S., Cavalcanti, A.B., Berwanger, O., Rosa, R.G., Veiga, V.C. et al Effect of dexamethasone on days alive and ventilator-free in patients with moderate or severe acute respiratory distress syndrome and COVID-19: the CoDEX randomized clinical trial. JAMA https://doi.org/10.1001/jama.2020.17021 (2020). 